\title{
Inhibition of O-GIcNAc transferase activity reprograms prostate cancer cell metabolism
}

\author{
Harri M. Itkonen ${ }^{1}$, Saurabh S. Gorad ${ }^{2,3}$, Damien Y. Duveau ${ }^{4}$, Sara E.S. Martin ${ }^{5}$, \\ Anna Barkovskaya2,7, Tone F. Bathen ${ }^{2}$, Siver A. Moestue ${ }^{2,3}$ and Ian G. Mills ${ }^{1,6,8}$ \\ ${ }^{1}$ Prostate Cancer Research Group, Centre for Molecular Medicine (Norway), University of Oslo and Oslo University Hospitals, \\ Gaustadalleen, Oslo, Norway \\ 2 Department of Circulation and Medical Imaging, NTNU, Trondheim, Norway \\ ${ }^{3}$ St. Olavs University Hospital, Trondheim, Norway \\ ${ }^{4}$ Division of Preclinical Innovation, National Center for Advancing Translational Sciences, National Institutes of Health, \\ Rockville, MD, USA \\ ${ }^{5}$ Department of Microbiology and Immunobiology, Harvard Medical School, Harvard Institutes of Medicine, Boston, MA, USA \\ ${ }^{6}$ Department of Molecular Oncology, Oslo University Hospitals, Oslo, Norway \\ 7 Department of Tumor Biology, Institute for Cancer Research, Radium hospital, Oslo University Hospital, Oslo, Norway \\ ${ }^{8}$ PCUK/Movember Centre of Excellence for Prostate Cancer Research, Centre for Cancer Research and Cell Biology (CCRCB), \\ Queen's University Belfast, Belfast, UK
}

Correspondence to: Harri M. Itkonen, email: harri.itkonen@ncmm.vio.no

Ian G. Mills, email: ian.mills@ncmm.vio.no ori.mills@qub.ac.uk

Keywords: androgen receptor, prostate cancer, metabolism, glycosylation, O-GlcNAc transferase

Received: August 10, $2015 \quad$ Accepted: January 19, $2016 \quad$ Published: January 27, 2016

\section{ABSTRACT}

Metabolic networks are highly connected and complex, but a single enzyme, O-GICNAC transferase (OGT) can sense the availability of metabolites and also modify target proteins. We show that inhibition of OGT activity inhibits the proliferation of prostate cancer cells, leads to sustained loss of c-MYC and suppresses the expression of CDK1, elevated expression of which predicts prostate cancer recurrence $(p=0.00179)$. Metabolic profiling revealed decreased glucose consumption and lactate production after OGT inhibition. This decreased glycolytic activity specifically sensitized prostate cancer cells, but not cells representing normal prostate epithelium, to inhibitors of oxidative phosphorylation (rotenone and metformin). Intra-cellular alanine was depleted upon OGT inhibitor treatment. OGT inhibitor increased the expression and activity of alanine aminotransferase (GPT2), an enzyme that can be targeted with a clinically approved drug, cycloserine. Simultaneous inhibition of OGT and GPT2 inhibited cell viability and growth rate, and additionally activated a cell death response. These combinatorial effects were predominantly seen in prostate cancer cells, but not in a cell-line derived from normal prostate epithelium. Combinatorial treatments were confirmed with two inhibitors against both OGT and GPT2. Taken together, here we report the reprogramming of energy metabolism upon inhibition of OGT activity, and identify synergistically lethal combinations that are prostate cancer cell specific.

\section{INTRODUCTION}

Prostate cancer is the most common male cancer in Europe and the USA. The androgen receptor (AR), a member of the nuclear hormone receptor family, is the major target in the treatment of the disease and its function is therefore the major focus for research. Enhanced AR activity promotes cell survival and proliferation by reprogramming tumour cell metabolism [1-3]. In addition, AR re-wires the metabolism of the normal prostate tissue, compared to the other differentiated cell and tissue types in the human body. The prostate gland secretes high levels of citrate, which is brought about by AR-regulated zinc accumulation to inhibit cis-aconitase [1]. This leads to 
decreased TCA cycle activity and ability to produce ATP. In the normal prostate tissue, AR additionally promotes the expression of lipogenic enzymes [4]. By doing this, AR alters the key energy producing and consuming mechanisms of the cell, and many of these changes are typically associated with proliferating cells.

In prostate cancer, the major traits of the metabolic phenotype are similar to what is seen in other cancers: increased glycolysis, abnormal phospholipid metabolism and dependence on reductive glucose metabolism [5]. It is highly likely that some of the metabolic features initially found in the normal prostate, are preserved in prostate cancer, and can be successfully targeted by repurposing metabolic inhibitors developed to treat other diseases. Indications that this is feasible have arisen from large epidemiological studies which, for example, have revealed that the use of the anti-diabetic drug metformin has strong effects on prostate cancer risk [6]. In addition, several novel compounds targeting cancer-specific metabolic abnormalities, such as glutaminase and monocarboxylate transporter 1 inhibitors, are currently assessed in clinical trials [7].

Metabolic pathways are highly inter-connected which complicates drug approaches, as normal cells can also be targeted. This challenge has led to the search for combinatorial treatment options to specifically target the vulnerabilities of cancer cells, which are not able to activate appropriate survival pathways upon dual targeting and do not cause toxicities in normal cells [8].

One of the key pathways sensing metabolic status in cells is the hexosamine biosynthetic pathway (HBP) [9]. HBP requires glucose, glutamine, acetate and UTP, and is thereby positioned to integrate information on the availability of nutrients. HBP produces the highenergy compound UDP-N-acetyl-D-glucosamine (UDPGlcNAc), which is a sugar donor involved in synthesis of other nucleotide sugars, complex glycosylation and also utilized by O-GlcNAc transferase (OGT) to modify target proteins via single sugar conjugation $[10,11]$. OGT targets key regulators of cell fate, including metabolic enzymes such as phosphofructokinase 1 [12], epigenetic regulators Oct4 and Sox2 [13] and transcriptional activity through glycosylation of the RNA Polymerase II tail to affect initiation complex formation [14]. Increased OGT expression has been detected in numerous cancers, including bladder cancer [15] and lung and colon cancers [16]. We recently reported that HBP enzymes are overexpressed in human prostate cancer patients and that three of the four HBP enzymes are induced by androgen stimulation $[2,17]$. All this has triggered great interest in developing small molecule inhibitors to target OGT [18, 19].

Given the established metabolic re-programming of prostate cancer cells, we hypothesised that inhibition of OGT activity might be able to distort cancer cell metabolism. In this study, we treated prostate cancer cells with a commercially available OGT inhibitor ST045849 and analysed samples with ${ }^{1} \mathrm{H}$ Nuclear Magnetic Resonance (NMR) spectroscopy. Treatment with the OGT inhibitor led to complete depletion of intracellular alanine. Gene expression analysis suggested that alanine was consumed by glutamic pyruvate transaminase 2 (alanine aminotransferase, GPT2). Combining OGT inhibition with GPT2 inhibition induced cell death specifically in prostate cancer cells.

\section{RESULTS}

\section{Establishment of OGT inhibitor dose that inhibits proliferation of cancer cells}

OGT activity is important for the proliferation of cancer cells, and inhibition of its activity inhibits tumour formation and metastasis $[17,20,21]$. These effects are mediated in part via down-regulation of oncogenic transcription factors such as c-MYC (Figure 1A). Previous studies have relied on transcriptomic profiling focusing on regulators of cell cycle genes. Given the prominent role of OGT as a metabolic integration point, we speculated that inhibition of its activity might additionally alter the metabolic profile of the cell.

First we wanted to identify an inhibitor dose that would modestly inhibit the proliferation of prostate cancer cells but ideally have no or low effects in cells representing normal prostate tissue. Therefore, we measured the viability of LNCaP (a prostate cancer cell line) and of PNT2 (a cell line representing normal prostate tissue) cells after 96 hours of treatment. This time-point was selected since prostate cancer cell proliferation rate is quite low and the effects on the metabolome should become clearer at late time-points. As expected, and based on previous studies [17], OGT inhibitor ST045849 significantly inhibited the viability of prostate cancer cells (Figure 1B). The same compound also decreased the viability of PNT2 cells, but to lower extent. We next evaluated the growth rate of both cell lines upon treatment with ST045849, and found that the higher dose $(40 \mu \mathrm{M})$ led to complete inhibition of proliferation in both prostate cancer cells and in cells representing normal prostate tissue (Figure 1C). Based on these data, we chose to treat cells with $20 \mu \mathrm{M}$ ST045849 to study the metabolic response of cells to OGT inhibition.

We next assessed if this dose was sufficient to decrease total O-GlcNAcylation in cancer cells, but observed that cells were able to compensate in the level of total protein O-GlcNAcylation at late time-points (data not shown), but at 1 hour after treatment with a low dose of ST045849 $(20 \mu \mathrm{M})$, we observed prominent decrease in the total protein O-GlcNAcylation (Suppl. Figure 1A). The observed compensation is not surprising, as it is well 


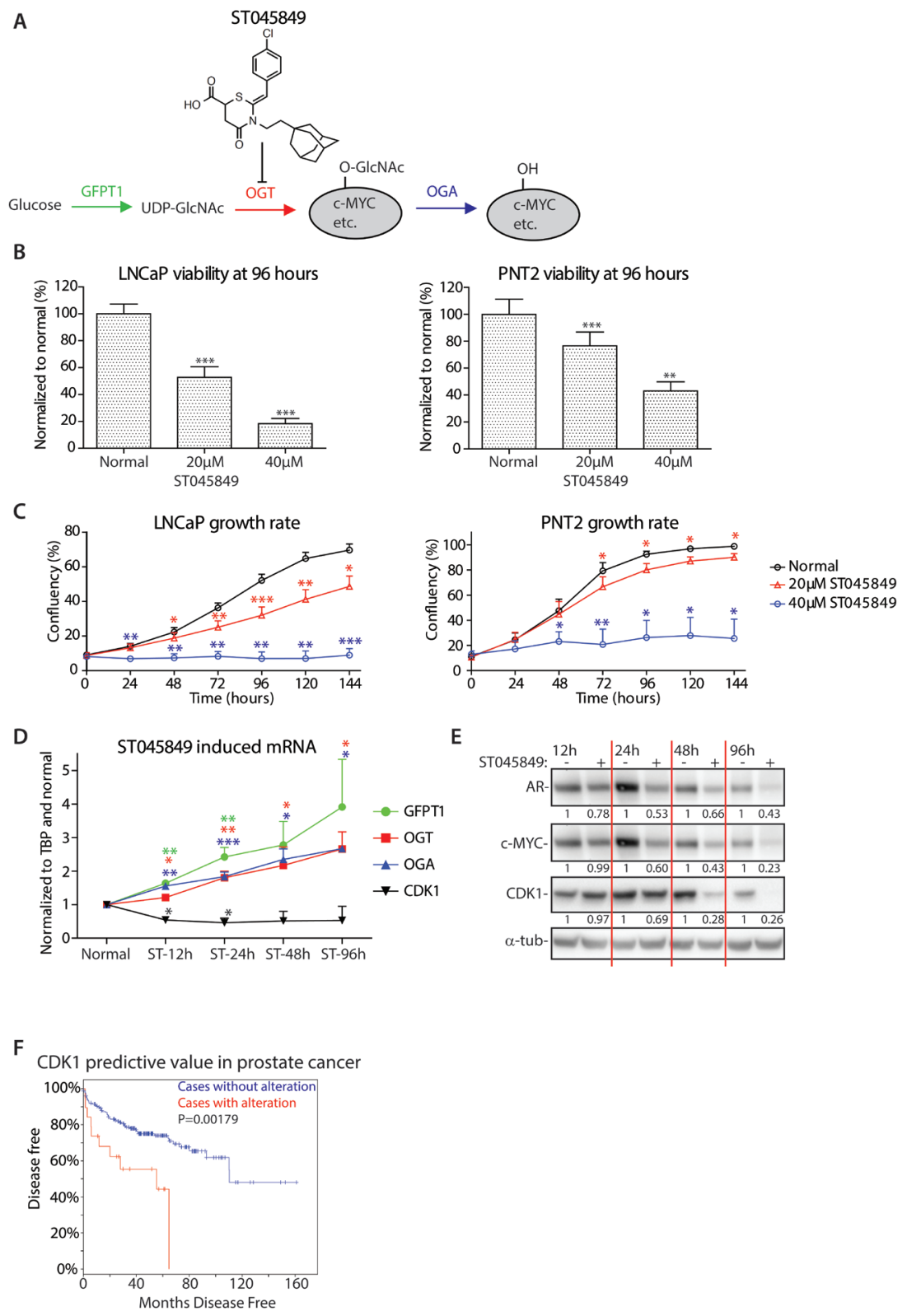

Figure 1: Effects of OGT inhibition on cell viability and establishment of the dose for metabolomics. A. Enzymes regulating O-GlcNAc cycling. GFPT1 (glutamine--fructose-6-phosphate transaminase 1) is the rate-limiting enzyme in the hexosamine (HBP) biosynthetic pathway and directs glucose to the HBP rather than glycolysis. UDP-GlcNAc (UDP-N-acetylglucosamine) is the end-product of HBP and is utilized by OGT to modify target proteins via single sugar conjugation. c-MYC is highlighted here as one of its targets. ST045849 is a small molecule inhibitor targeting OGT. OGA (N-Acetyl-Beta-D-Glucosaminidase) removes O-GlcNAc from target proteins. B. LNCaP and PNT2 cells were treated with the indicated doses of OGT inhibitor ST045849 for 96 hours, and the viability was determined with the CellTiter-Glow ${ }^{\circledR}$ (CTG) assay. The data shown is an average of four biological replicates and Standard Error of the Mean (SEM) is shown. The significance was assessed with Student's $t$-test, $* *<0.01$ and $* * *<0.001$. C. Growth rate of cells after indicated treatments. The data shown is an average of four biological replicates with SEM. The significance was assessed with Student's $t$-test as above, $*<0.05, * *<0.01$ and $* * *<0.001$, red stars indicate comparison between normal and $20 \mu \mathrm{M} \mathrm{ST045849}$ and blue stars

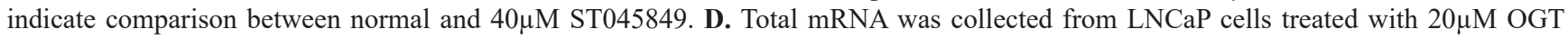
inhibitor ST045849 for 12, 24, 48 and 96 hours, and analysed with RT-qPCR. OGT inhibitor treated samples were normalized to sample without treatment at 12 hours. The data shown is an average of at least three biological replicates with SEM. The significance was assessed with Student's $t$-test $(*<0.05, * *<0.01$ and $* * *<0.001)$ by comparing untreated sample from the corresponding time point, and each colour corresponds to the transcript measurement highlighted with the same colour. E. Protein lysates were harvested at 12, 24, 48 and 96 hour time-points and blotted for the markers of interest. The data shown is representative of three biological replicates. Densitometry was used to quantitate the intensity of each band, AR, c-MYC and CDK1 intensities were normalized to loading control and un-treated sample from each time-point was set to 1 . F. Potential correlation of CDK1 expression with biochemical recurrence in prostate cancer patients was assessed using cBioPortal for Cancer Genomics (http://www.cbioportal.org/) using Taylor \& al. data set[25]. Increased expression of CDK1 predicts biochemical recurrence with $\mathrm{p}$ value of 0.00179 . 
established that O-GlcNAcylation is tightly regulated $[17,22]$. This compensation was mediated, at least in part, through increased expression of the rate-limiting enzyme in the hexosamine biosynthetic pathway, GFPT1 (Figure 1D and Suppl. Figure 1C, 1D). Interestingly, the expression of both OGT and O-GlcNAcase (OGA) were increased in the mRNA but not noticeably in protein level upon OGT inhibitor treatment. We next assessed whether some of the effects previously reported upon treatment with ST045849 were seen at a dose of $20 \mu \mathrm{M}$, and observed that the compound led to sustained decrease of c-MYC at the 24, 48 and 96 hour time-points (Figure 1E) [17]. In addition, we assessed the expression levels of CDK1, the gene found to be most down-regulated by ST045849 treatment [17]. The expression of CDK1 was suppressed at 12 hours and this decreased expression was maintained until 96 hours, and the same was true in protein level (Figure 1D, 1E).

A novel OGT inhibitor, OSMI-1, was recently reported with higher specificity against OGT than other extant compounds $[18,19]$. In an in vitro assay utilizing purified OGT, OSMI-1 has a 20-fold lower $\mathrm{IC}_{50}$-value once compared to ST045849. OSMI-1 has fewer side effects, and compound appears not to affect plasmamembrane glycosylation, but still requires reasonably high doses to induce effects on the total-O-GlcNAc $(50 \mu \mathrm{M}$ for maximal inhibition) [19]. We first confirmed that OSMI-1 decreased total-O-GlcNAc (Suppl. Figure 1E). Treatment with OSMI-1 led maximally to $60 \%$ decrease in CDK1 mRNA (Suppl. Figure 1F). Importantly, and in agreement with ST045849-data, OSMI-1 decreased both c-MYC and CDK 1 proteins by $40 \%$ at 24 and 48 hours after the treatment (Suppl. Figure 1G). CDK1 phosphorylates AR and thereby stabilizes the protein and protein's transcriptional output [23]. As expected based on the reported CDK1 function, OGT inhibition also decreased AR protein expression (Figure 1E and Suppl. Figure 1G).

So far, we have established an inhibitor dose that displayed a clear decrease in the expression of an important cell cycle regulator, CDK1 [24], and a decrease in the expression of $\mathrm{AR}$, a major drug target in prostate cancer. Analysis of a published prostate cancer microarray data set [25] revealed that increased expression of CDK1 predicts prostate cancer recurrence after surgery with high significance ( $p=0.00179$, Figure $1 F)$. Based on these data, we decided to analyse the possible metabolic adaptations that enable prostate cancer cell survival despite the significant down-regulation of prominent prostate cancer oncogenes, c-MYC and AR.

\section{Inhibition of O-GIcNAc transferase activity inhibits glycolysis}

Having established a dose of OGT inhibitor ST045849 for metabolic profiling, we used ${ }^{1} \mathrm{H}$ NMR spectroscopy to analyse cell culture media of LNCaP prostate cancer cells treated with the OGT inhibitor. In accordance with growth inhibition, we observed a decrease in glucose consumption and in lactate production, potentially reflecting the inhibitory effects on cell growth (Figure 2A). However, we speculated that the treatment imposed a selection pressure on prostate cancer cells for a switch in metabolic dependency. Since oxidative phosphorylation can be sustained by other substrates than glucose we hypothesised that the decreased ability of these cells to cope with lower glucose uptake should make them sensitive to inhibitors of mitochondrial respiration. In order to test this hypothesis, we used two compounds: (1) a highly potent mitochondria complex 1 inhibitor (rotenone) at a dose of $10 \mathrm{nM}$ which leads to $80 \%$ decrease in complex 1 activity [26] but has only modest effect on viability, and (2) metformin (used at a $1 \mathrm{mM}$ concentration), another complex 1 inhibitor with less specificity but used in clinical setting [27]. Treatment of LNCaP cells with rotenone or metformin alone led to $20 \%$ $40 \%$ decrease in cell viability, while combining either of the compounds with the OGT inhibitor led to $80 \%$ decrease in viability (Figure 2B). We also observed near complete growth inhibition upon combinatorial treatment (Figure 2C and 2D). Interestingly, while both rotenone and metformin modestly decreased the viability and growth rates of PNT2 cells, we did not observe any additive effects with OGT inhibitor (Figure 2B-2D). These results were confirmed with the novel OGT inhibitor OSMI1 , and combinatorial treatments with either rotenone or metformin statistically significantly decreased the viability and blocked proliferation of prostate cancer cells but had no effect on cells representing normal prostate tissue (Suppl. Figure 2A-2C). In addition, treatment of another prostate cancer cell line, PC3, with either of the OGT inhibitors together with rotenone or metformin statistically significantly reduced the viability of cells (Suppl. Figure 2D).

Extracellular metabolite concentrations can reflect the energetic status of the cell, as shown here by identifying potential combinatorial treatments to inhibit proliferation. However, measuring intracellular metabolites provides a more detailed picture of the cellular metabolic networks.

\section{Inhibition of O-GlcNAc transferase activity leads to depletion of intracellular alanine}

Having established that glucose consumption is significantly decreased in prostate cancer cells treated with OGT inhibitor ST045849, we moved on to evaluate the levels of intracellular metabolites. We noted prominent accumulation of glucose and a decrease in intracellular lactate concentration, in support of decreased glycolysis (Figure 3A).

Interestingly, intra-cellular alanine was almost completely depleted in LNCaP cells and glutamate was 
A

LNCaP media: example NMR-spectra

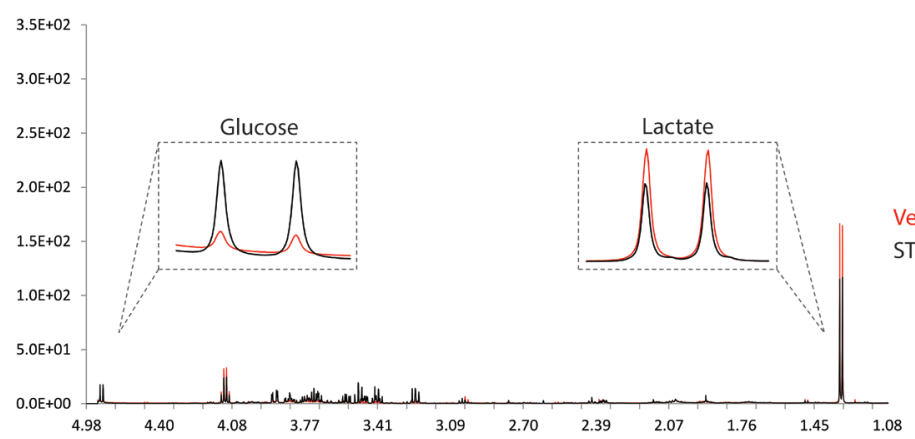

Extra-cellular metabolites

Glucose

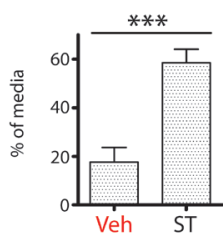

ST045849

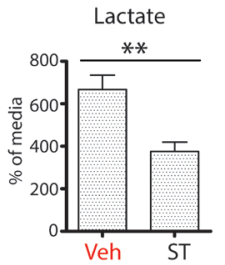

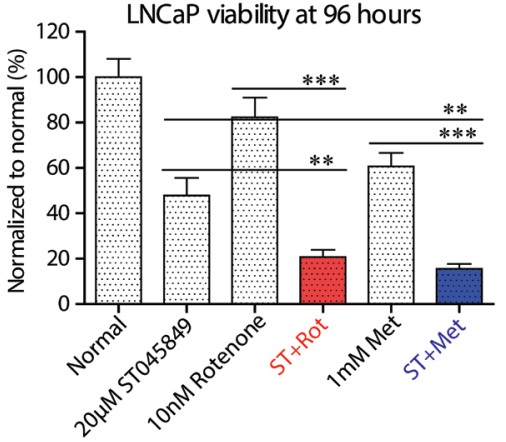

C

LNCaP growth rate

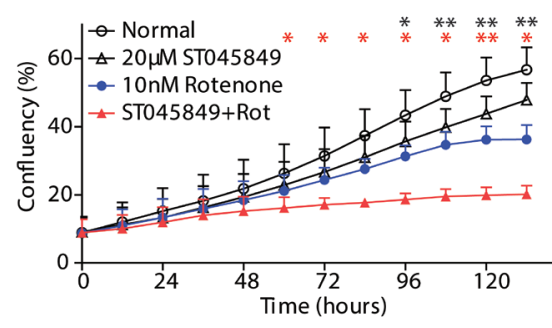

D

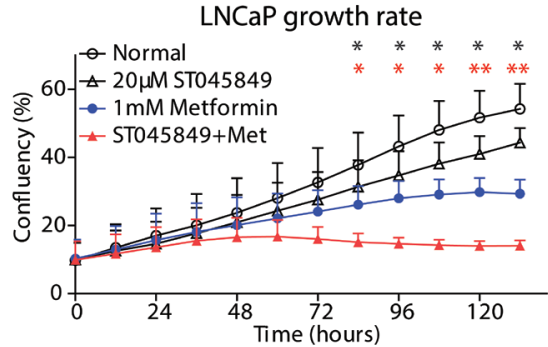

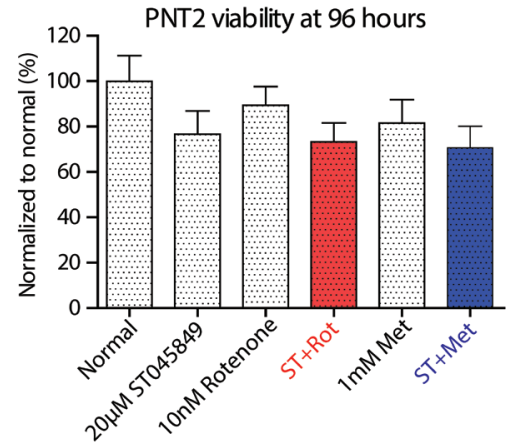

PNT2 growth rate

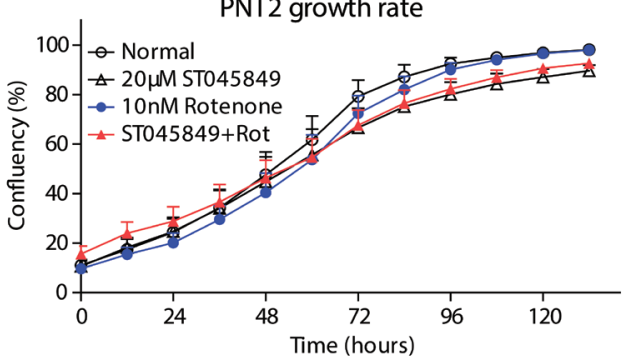

PNT2 growth rate

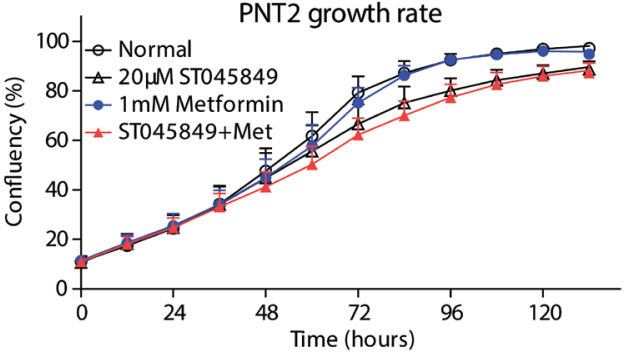

Figure 2: Metabolomic profiling of cell culture media after OGT inhibitor ST045849 treatment. A. LNCaP cells were treated with $20 \mu \mathrm{M}$ OGT inhibitor ST045849 for 96 hours, cell media were collected and analyzed by ${ }^{1} \mathrm{H}$ NMR. An example of the obtained NMR spectra is shown. The levels of glucose and lactate were determined from the cell culture media by ${ }^{1} \mathrm{H}$ NMR. The data shown is an average of seven biological replicates with SEM. The significance was assessed with Student's $t$-test, $* *<0.01, * * *<0.001$. B. Cells were treated as indicated in the figure and the viability of cells was analysed with CTG reagent after 96 hours treatment. Viability of the untreated sample was set to $100 \%$ and treatments were normalized to this. The data shown is an average of four biological replicates with SEM. The significance was assessed with Student's $t$-test, $* *<0.01, * * *<0.001$. C. and D. Cells were treated as indicated in the figure and the growth rate of cells was recorded by life cell imaging. The data shown is an average of four biological replicates with SEM. The significance was assessed with Student's $t$-test, $*<0.05$ and $* *<0.01$. Black stars indicate comparison between rotenone (or metformin) only and combination of ST045849 with rotenone (or metformin), while red stars indicate comparison between ST045849 and combinatorial treatments. 
decreased. Alanine and glutamate are inter-convertible, non-essential amino acids. At the systemic level, alanine is part of the alanine cycle, which transports carbon skeletons between muscle cells and liver, to signal for the need of increased glucose release [28]. Glutamic pyruvate transaminase (GPT2, alternative name: alanine aminotransferase) is the enzyme catalysing the metabolism of alanine (and of $\alpha$-ketoglutarate) to produce pyruvate and glutamate (Figure 3B). Pyruvate can be utilized to support the TCA cycle and ATP production. In this context, alanine can be considered as an alternative energy source in the absence of sufficient glycolysis. In support of this, GPT2 is induced by starvation (Suppl. Figure 3A and 3B).

OGT inhibition increased the expression of GPT2 already at 12 hours and increased expression was maintained until 96 hours (Figure 3C). Interestingly, the intracellular alanine level was decreased by $50 \%$ after 48 hours (Suppl. Figure 3C), and the amino acid was almost completely consumed after 96 hours in LNCaP cells (Figure 3A). mRNA and metabolomics data support the hypothesis that GPT2 is activated in order to consume alanine. We next used a GPT2 activity assay to confirm that the enzyme activity is increased (Figure 3D). OGT inhibition doubled GPT2 activity, when compared to the untreated control.

Taken together, these results suggest that OGT inhibition triggers cells to rely on alanine as an alternative energy source and highlight an important role for the GPT2 enzyme.

\section{Inhibition of alanine aminotransferase has additive effect with OGT inhibitor}

OGT inhibition led to a decrease in glucose consumption and to a significantly higher alanine consumption. In order to test whether GPT2 activity is important for cell survival upon OGT inhibition, we used two GPT2 inhibitors: chloro-alanine (Cl-alanine) and cycloserine. Combination of either of the two OGT inhibitors (ST045849 or OSMI-1) with either of the two GPT2 inhibitors led to a statistically significant decrease in the viability of LNCaP cells (Figure 4A). Both OGT inhibitors sensitized another prostate cancer cell line, $\mathrm{VCaP}$, to cyclo-serine (Figure 4B). In addition, OGT inhibition sensitized a third prostate cancer cell line (PC3) to GPT2 inhibition Suppl. Figure 4A and 4B). We next confirmed the small molecule data by inhibiting OGT expression with siRNAs and treated cells with Cl-alanine (Suppl. Figure 4C). This combinatorial treatment strategy also completely blocked proliferation of LNCaP cells and inhibited proliferation of PC3 cells (Suppl. Figure 5A-5C).

We next evaluated the combinatorial treatment strategy of simultaneous targeting of OGT and GPT2 in PNT2 cells, and observed that both Cl-alanine and cycloserine alone decreased cell viability, but no additive effect was observed when both GPT2 and OGT inhibitors were combined (Figure 4C). However, combinatorial treatment decreased the growth rate of PNT2 cells, albeit to a lesser extent than LNCaP cells (Suppl. Figure 5A).

We hypothesised that a cell line derived from normal prostate epithelium might be able to slow down proliferation due to acute deficiency in energy production, while a cell death response would be activated in prostate cancer cells. This would explain the discrepancy observed between the viability and the growth rate data. In order to test this hypothesis, we applied our combinatorial treatments to LNCaP and PNT2 cells and evaluated caspase 3/7 activation in real-time. Interestingly, caspase activation was prominently enhanced by the combinatorial treatment with OGT inhibitors and GPT2 inhibitors in LNCaP cells but not in PNT2 cells (Figure 5). Of high importance, the clinically approved drug, cycloserine, was more effective in activating the combinatorial cell death response.

\section{DISCUSSION}

In this study, we used ST045849, a commercially available inhibitor, to study the effect of OGT inhibition on prostate cancer cell viability and metabolic reprogramming. We first established a compound dose that significantly limited the proliferation of prostate cancer cells (Figure 1B and 1C). We observed decrease in the total-O-GlcNAc levels at 1 hour after the treatment (Suppl. Figure 1A). However, total O-GlcNAc levels varied from experiment-to-experiment over time points in all conditions tested which may reflect the complex role of the HBP as a sensor of changes and stresses across a range of metabolic processes. Some of this may also at the longer time points reflect an adaptation to maintain the levels of total-O-GlcNAcylation, through increased expression of GFPT1 (Figure 1D and Suppl. Figure 1C and 1D).

Consequently, in order to understand the impact of targeting OGT on cell growth we needed to look beyond total O-GlcNAc levels and down to effects on specific genes and proteins. We speculate that proteins having only single or few sugar conjugations (such as c-MYC [29]) will show regulatory response at low doses of OGT inhibitor but this is not evident in the level of total O-GlcNAc. OGT regulates target proteins according to nutrient availability $[10,30]$ and growth-promoting factors need to be shut-down in the absence of nutrients to avoid cell death. However, OGT additionally has house-keeping target(s), amongst which nucleoporins (NUPs) are highly abundant [31, 32]. Complete block of OGT activity leads to decreased O-GlcNAcylation and thereby ubiquitinylation and degradation of NUPs, which compromises the nuclear pore selectivity filter [31]. Certain functions of OGT are important for the survival of normal cells, and one option to position OGT as a drugtarget, is to use low dose of inhibitor and try to identify 
A
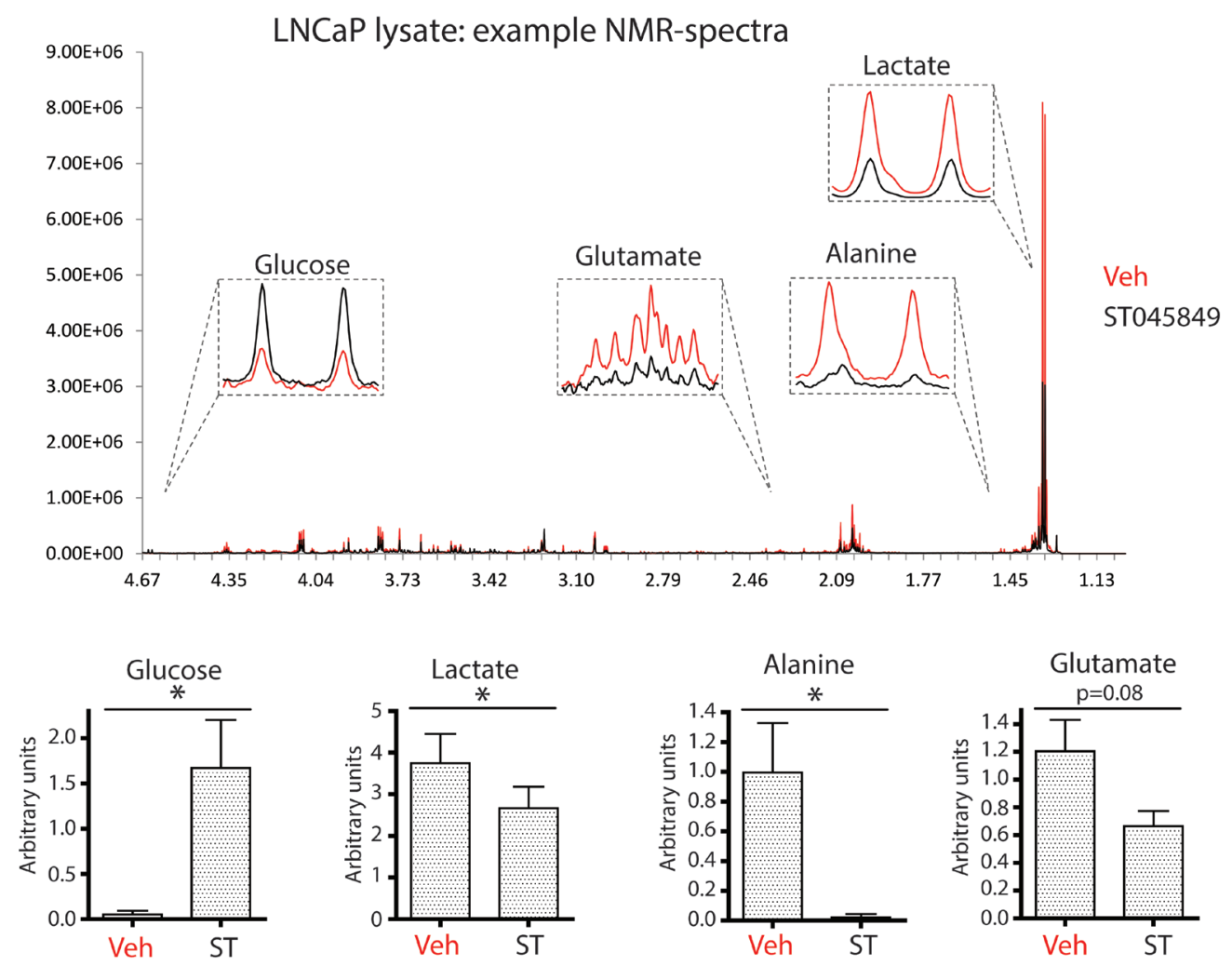

B Alanine $+\alpha$-ketoglutarate $\stackrel{\mathrm{GPT} 2}{\longrightarrow}$ Glutamate + pyruvate

C

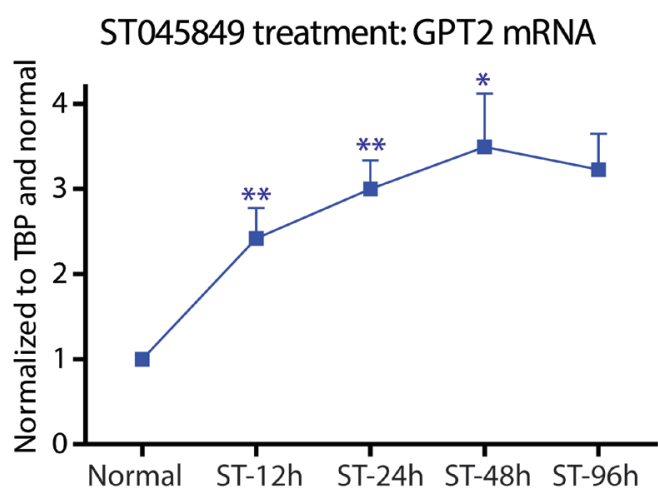

D

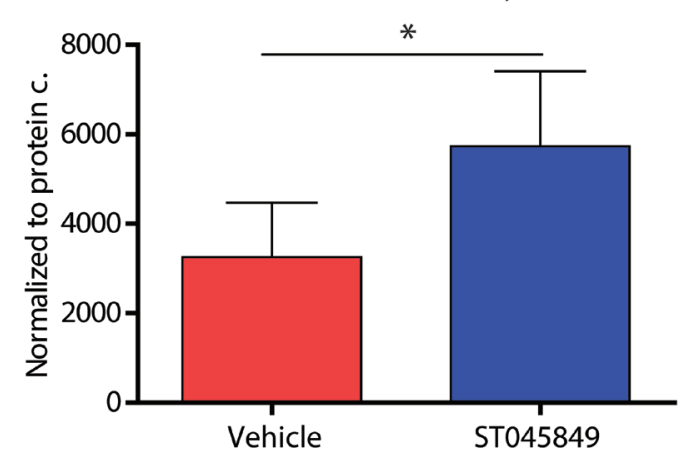

Figure 3: Effects of OGT inhibitor ST045849 on the levels of intracellular metabolites and enzyme activity. A. LNCaP cells were treated with OGT inhibitor ST045849 for 96 hours, cell lysates were collected and analysed by ${ }^{~} \mathrm{H}$ NMR. An example of the obtained NMR spectra is shown. Quantitation of the ${ }^{1} \mathrm{H}$ NMR data. The data shown is an average of seven biological replicates with SEM. The significance was assessed with Student's $t$-test, $*<0.05$. B. Enzymatic reaction catalysed by GPT2 (glutamic pyruvate transaminase). C. Total mRNA was collected from cells treated with OGT inhibitor ST045849 for 12, 24, 48 and 96 hours, and analysed with RT-qPCR. OGT inhibitor ST045849 treated samples were normalized to sample without treatment at 12 hours. The data shown is an average of at least three biological replicates with SEM and significance was assessed by comparing untreated sample from the corresponding time point, $*<$ $0.05, * *<0.01$. D. Glutamic pyruvate transaminase (GPT2) assay performed from cell lysates treated either with a vehicle or with $20 \mu \mathrm{M}$ of OGT inhibitor ST045849. The GPT2 activity was assessed at 48 hours. The data shown is an average of four biological replicates with SEM. The significance was assessed with Student's $t$-test, $*<0.05$. 
A

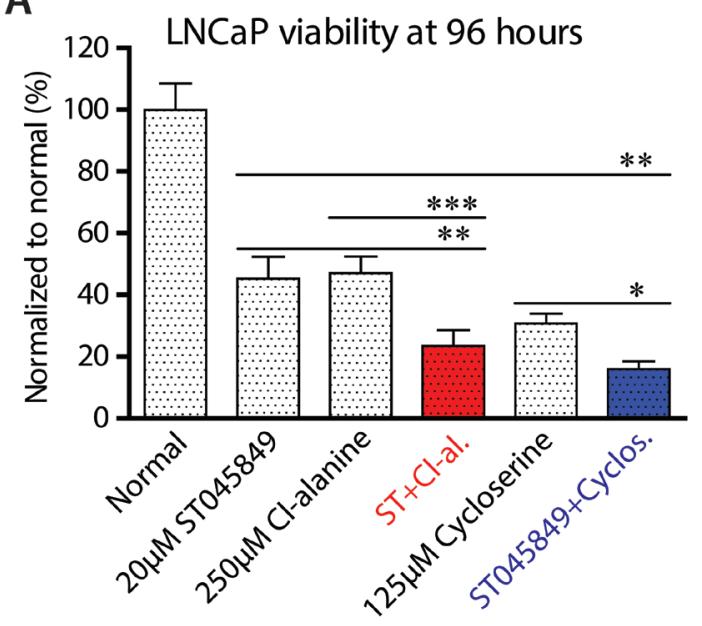

B

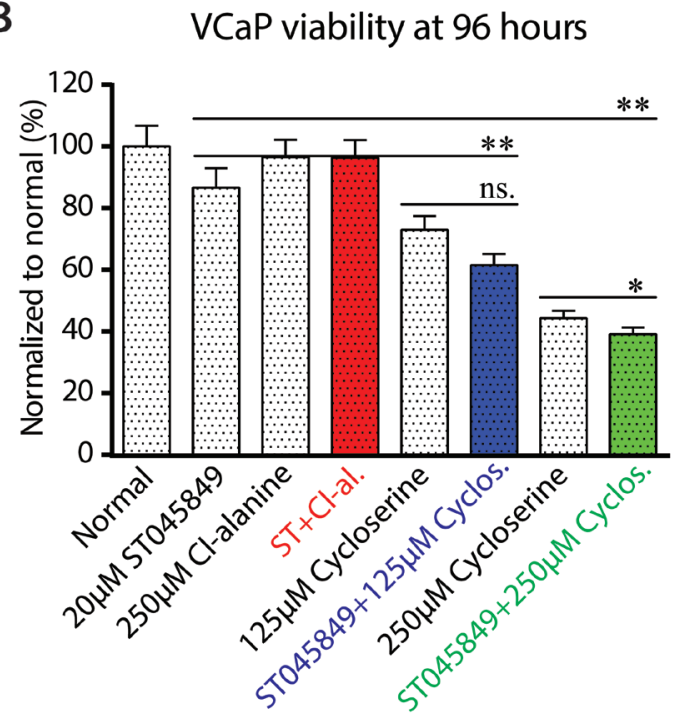

C

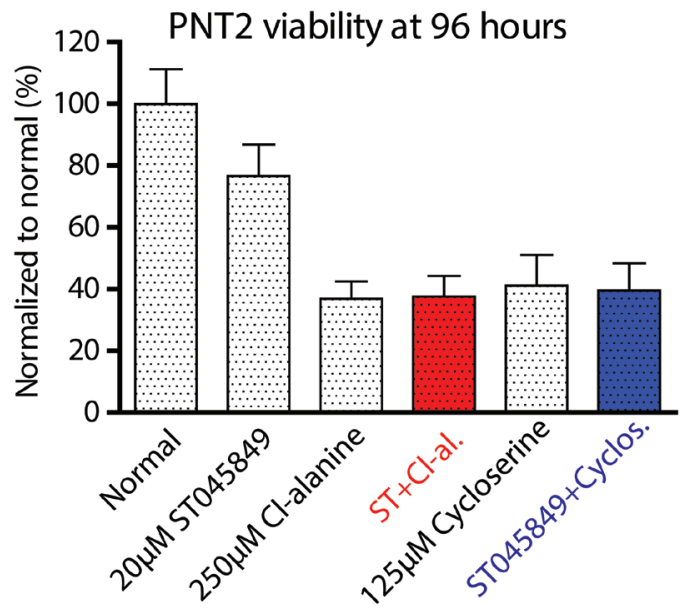

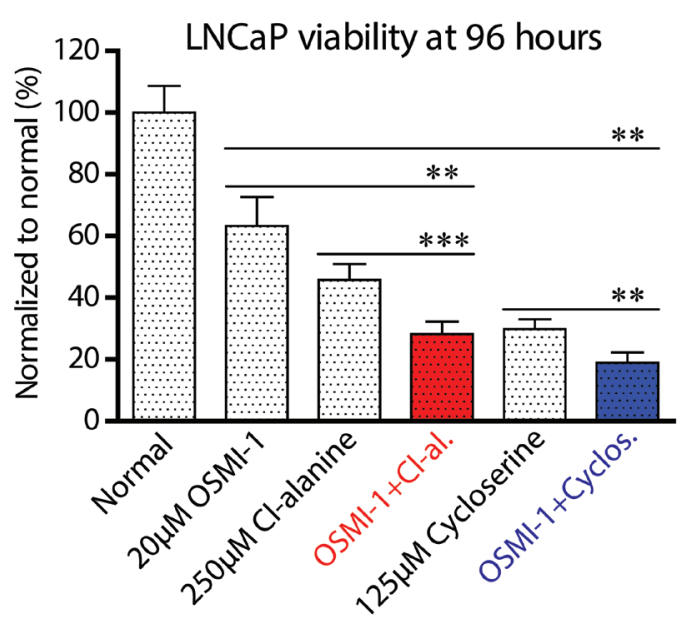

VCaP viability at 96 hours
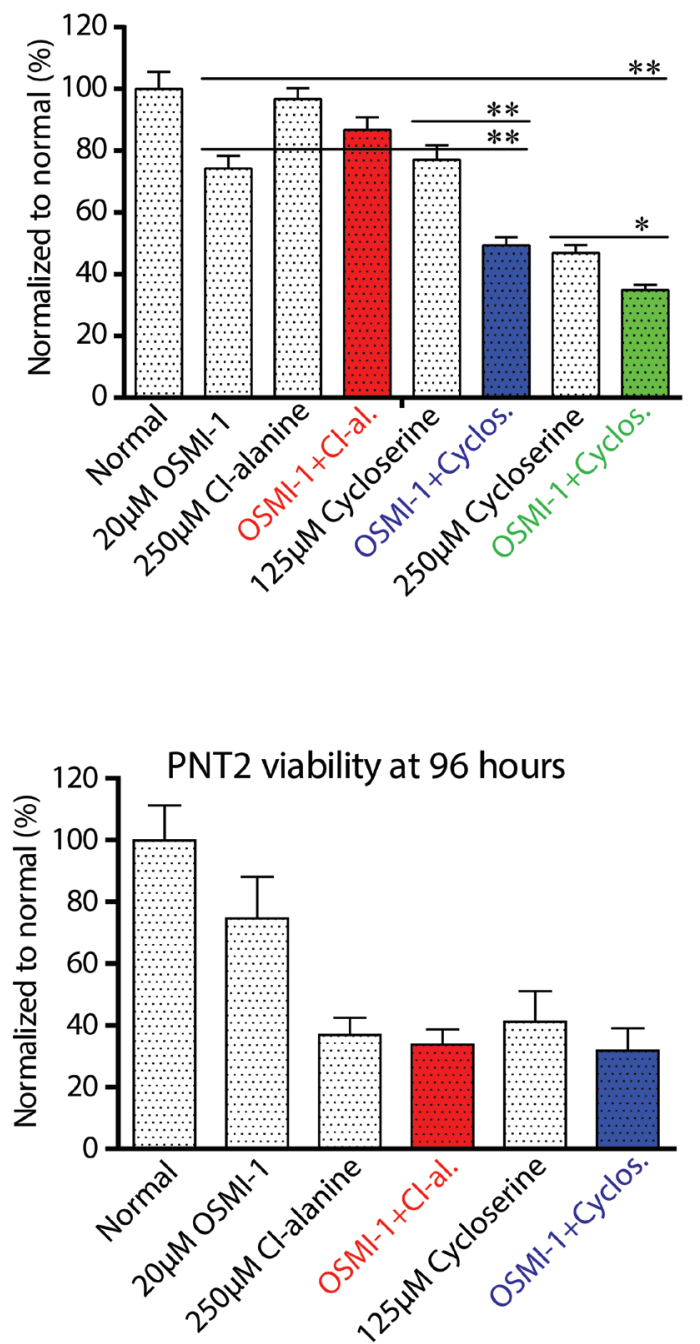

Figure 4: Viability of cells after combinatorial treatment with O-GIcNAc transferase and alanine aminotransferase inhibitors. A., B. and C. Cells were treated as indicated in the figure and the viability of cells was assessed after treatment with OGT inhibitor ST045849 or OSMI-1 alone or in combination with glutamic pyruvate transaminase (GPT2) inhibitors Cl-alanine or cycloserine. The data shown is an average of four biological replicates with SEM and significance was assessed with Student's $t$-test, $*<0.05,{ }^{*}<<$ 0.01 and $* * *<0.001$. 
cancer-cell specific vulnerabilities.

In this work, we observed that low dose of ST045849 and OSMI-1 treatment was sufficient to decrease the expression of AR, c-MYC and CDK1 (Figure $1 \mathrm{D}$ and $1 \mathrm{E}$ and Suppl. Figure $1 \mathrm{~F}$ and $1 \mathrm{G})$. Inhibition of OGT expression has been shown to decrease the viability and invasion of prostate cancer cells through reduction of transcription factor FoxM1 [21]. Interestingly, CDK1 is required for FoxM1 transcriptional activity during cell cycle progression $[33,34]$. It appears that decrease OGT activity can disrupt this positive cell cycle promoting regulatory relationship in prostate cancer cells and it remains to be determined if same is true in other cancers.

Increased CDK1 expression has not been correlated with disease progression in prostate cancer, and we show that its elevated expression increases the likelihood of prostate cancer recurrence (Figure 1F). One of the major functions of CDK1 is cell cycle regulation [24], while
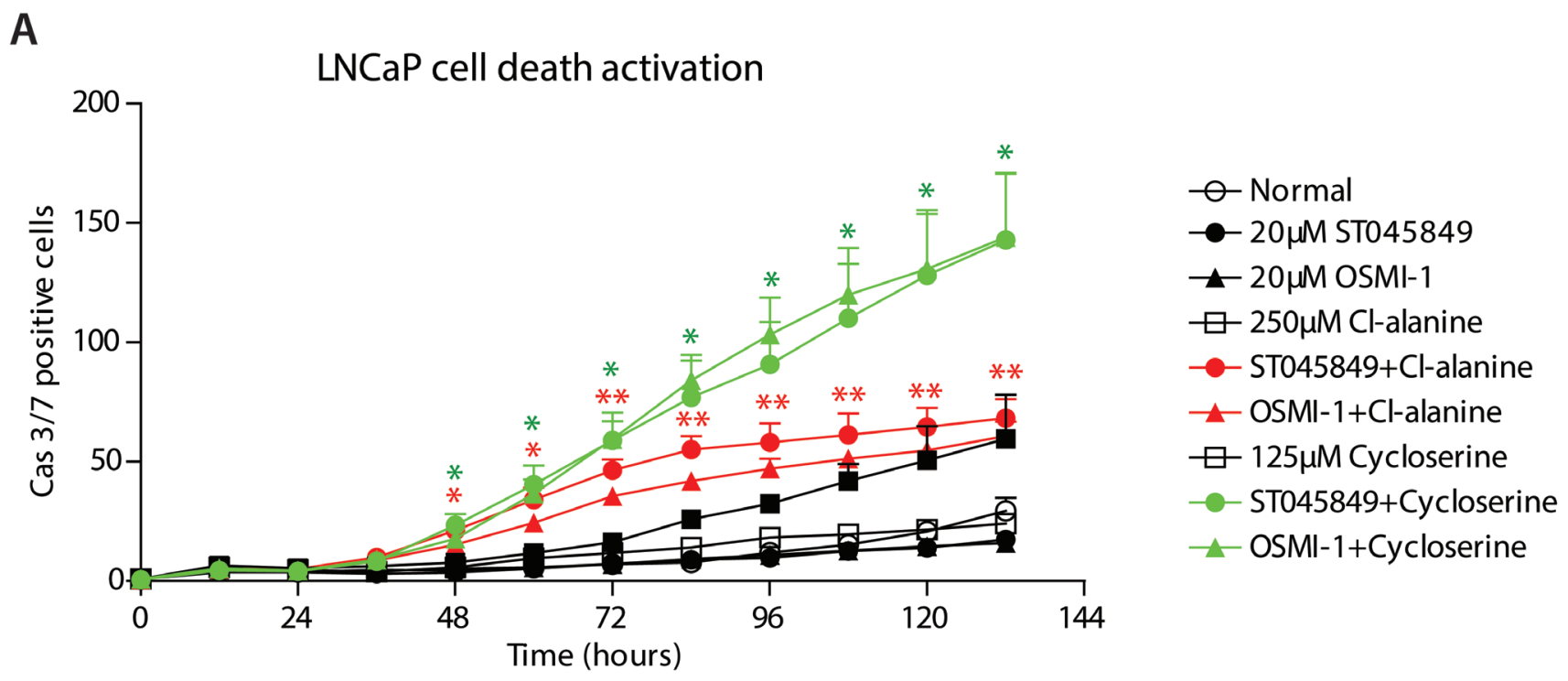

B

\section{PNT2 cell death activation}

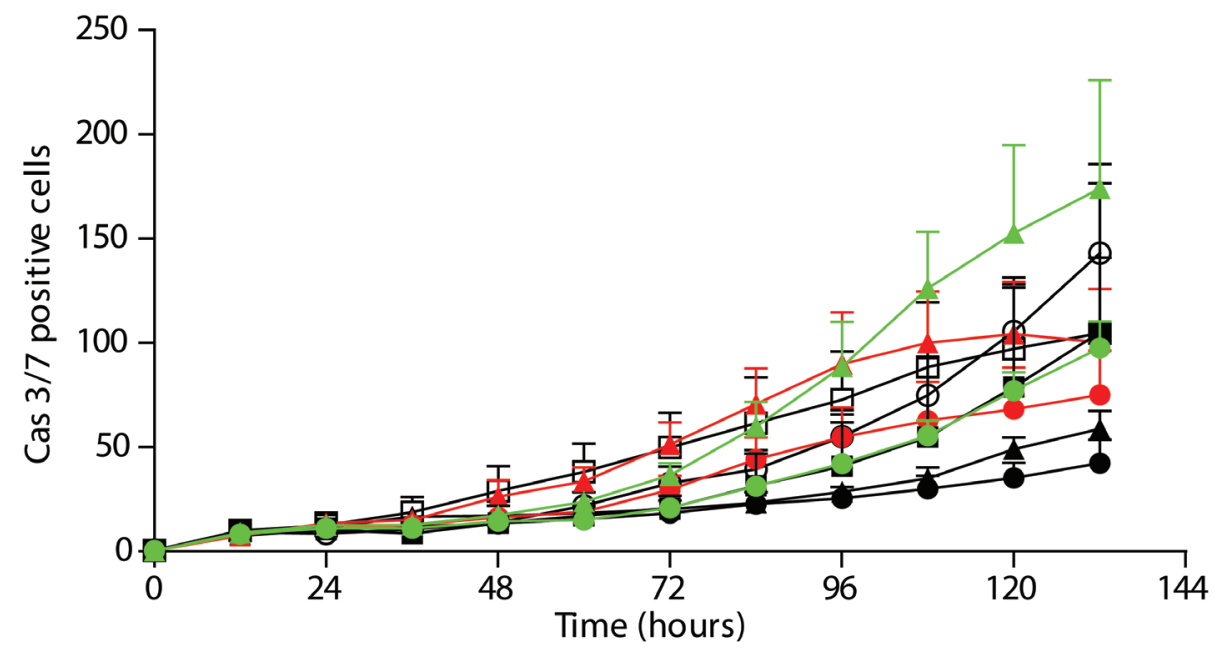

Figure 5: Combinatorial treatment with O-GIcNAc transferase inhibitors and alanine aminotransferase inhibitors activates cell death response in prostate cancer cells. A. and B. Activation of caspases 3 and 7 was assessed in real time, imaging every 12 hours. The data shown is an average of four biological replicates with SEM and significance was assessed with Student's $t$-test, *< 0.05 and $* *<0.01$. Red stars indicate comparison between either of the OGT inhibitors (ST045849 or OSMI-1) combined with Cl-alanine against any of the single treatments, while green stars indicate comparison between either of the OGT inhibitors (ST045849 or OSMI-1) combined with cycloserine against any of the single treatments. 
OGT itself directly acts on the key cell cycle regulators such as HCF-1 [35] and DNA damage response proteins [36]. OGT inhibition might sensitize cancer cells to DNA damage inducing agents, and this approach clearly demands further research.

We next performed metabolic profiling of prostate cancer cells after OGT inhibitor treatment. Treatment with ST045849 led to decreased glucose consumption and lactate production (Figure 2A). This can, at least in part, be explained through increased expression of GFPT1, to divert glucose into hexosamine biosynthetic pathway (Figure 1D). These data suggest that cancer cells decrease glycolysis due to OGT inhibition, and might be sensitized to inhibitors of oxidative phosphorylation. We confirmed this by treating cells with two different OGT inhibitors (ST045849 and OSMI-1) and with two inhibitors of oxidative phosphorylation, and observed cancer cell specific growth blockage (Figure 2B- 2D and Suppl. Figure 2).

We believe that small molecule inhibitors represent the most effective therapeutic strategy to translate these findings into the clinic, even though we did observe a clear adaptation to the inhibitor treatment through increased expression of GFPT1 (Figure 1D). However, some of the desirable effects obtained through OGT inhibition, such as decrease in c-MYC, AR and CDK1, were maintained (Figure 1D and 1E).

OGT inhibition caused a near-to-complete depletion of alanine in LNCaP cells (Figure 3A). Alanine is likely consumed by GPT2 as an adaptation to OGT inhibition. Interestingly, in the absence of any inhibitor, prostate cancer cells accumulate alanine [37], and alanine level is also increased in primary samples of prostate cancer [38]. Recent advances in magnetic resonance imaging (MRI) technology may allow non-invasive, real-time assessment of alanine metabolism through hyperpolarized ${ }^{13} \mathrm{C}$ labelled pyruvate, which is converted to lactate and alanine in prostate cancer[39].

GPT2 can be inhibited with small molecule inhibitors Cl-alanine and cycloserine [40]. Cycloserine is an FDA-approved drug to treat resistant forms of tuberculosis[41], but the drug also inhibits human GPT2, as shown previously [40] at similar doses as used in our study. Combinatorial treatments strongly inhibited cell viability and activated cell death response in prostate cancer cells but not in cells representing normal prostate tissue (Figure 4 and 5). Notably, GPT2 activity assays have been developed and applied successfully to blood samples [42].

In this work, we have defined complementary inhibitor targets, oxidative phosphorylation and GPT2, which can enhance response to OGT inhibition in prostate cancer cells. FDA-approved drugs such as metformin and cycloserine are available in clinical practice, thereby offering accelerated translation of these findings. OGT is also of high importance in normal cells [43], and combinatorial targeting of OGT and cancer-cell specific metabolic abnormalities may allow significant dose reduction. GPT2 functions to sustain pyruvate levels and mitochondrial activity, which enables non-invasive therapy monitoring using hyperpolarized ${ }^{13} \mathrm{C}$-pyruvate MRI. The next steps in taking OGT as a drug target into the clinic requires utilization of model organisms to better understand potential toxicities and the success of combinatorial treatment regimes.

\section{MATERIALS AND METHODS}

\section{Cell lines, maintenance and treatments}

Cells were obtained from ATCC and were maintained according to ATCC guidelines. For metabolomic profiling, cells were plated into either media with a commercial OGT inhibitor (ST045849, TimTec) or a vehicle control (DMSO). O-(2-Acetamido2-deoxy-D-glucopyranosylidenamino) N-phenylcarbamate (PUGNAc), metformin, rotenone, cycloserine and $\mathrm{Cl}-$ alanine were obtained from Sigma. OSMI-1 compound was a gift from Professor Suzanne Walker (Harvard Medical School). Cells were allowed to attach for 1 to 3 days, and then treated as indicated in the beginning of the experiment (media was not changed at any point). OGT knockdown was performed with Lipofectamine RNAiMAX reagent according to manufacturer's instructions (Invitrogen) and OGT targeting siRNAs were obtained from Lifetechnologies (s16094 and s16095).

\section{Analysis of viability, growth rate and GPT2- and caspase-activity}

Cells were plated into 384-well plates one day before treatment, unless otherwise stated. Viability was determined with cell titer glow (CTG) reagent (Promega). Caspase activation (CellPlayer reagent) and growth rates were determined with the IncuCyte instrument (EssenBiosciences). For GPT2 assays, two million cells were plated and activity was assessed with ALT Activity Assay (Sigma).

\section{Prepation of cell lysates}

For NMR profiling, 350000 cells were plated into three 6-well plate wells, and combined for analysis. Media was snap-frozen with liquid nitrogen and stored in $-80^{\circ} \mathrm{C}$ until the analysis. Cells were collected into ethanol $\left(-20^{\circ} \mathrm{C}\right)$. After this, cell lysates were centrifuged (18 000rpm, 10 minutes), soluble material was transferred to a new tube, evaporated with a speedvac and stored in $-80^{\circ} \mathrm{C}$ until analysis. For mRNA profiling, mRNA was 
collected with illustraMiniSpin kit (GE Healthcare), reverse-transcribed with (qScript cDNA Synthesis Kit, Quanta Biosciences), and qPCR was performed with primers designed by the Primer3.0 tool (Supplementary Table 1).

\section{Preparation of cell lysates for western blotting}

Cell lysates were prepared as described previously[17] and cell lysis buffer was supplemented with protease and phosphatase inhibitors (Roche) and PUGNAc. Membranes were probed with antibodies against AR (SantaCruz N-20 sc-816), c-MYC (Abcam ab32072), CDK1 (CST-9116), GFPT1 (CST-3818), OGT (CST- 5368), OGA (Sigma HPA036141), $\alpha$-Tubulin (Millipore CP06) and GAPDH (CST-2118S).

\section{NMR profiling}

Cell extracts were dissolved in $600 \mu \mathrm{lBS} / \mathrm{D} 2 \mathrm{O}$ solution containing $1.11 \mathrm{mM}$ trimethylsilyl propionic acid (TSP) as a chemical shift reference. $500 \mu$ l of culture medium and blank culture medium samples $(n=2)$ were mixed with $100 \mu \mathrm{l}$ PBS/D2O/TSP solution. The samples were transferred to $5 \mathrm{~mm}$ NMR tubes (Bruker Biospin $\mathrm{GmbH}$, Germany) for NMR analysis at the MR Core Facility, NTNU, Trondheim, Norway, using a Bruker Avance III Ultrashielded Plus $600 \mathrm{MHz}$ spectrometer (Bruker Biospin $\mathrm{GmbH}$ ). The spectrometer was equipped with a $5 \mathrm{~mm}$ QCI Cryoprobe. Proton spectra were acquired using 1D NOESY (Bruker: noesygppr1d) with presaturation and spoiler gradients as described previously [44]. A standard $1 \mathrm{mM}$ creatine reference solution was analyzed under identical experimental conditions and used as an external calibration standard. Pre-processing of NOESY spectra was performed using TopSpin 3.2 (Bruker BioSpin GmbH, Ettlingen, Germany). After $0.30 \mathrm{~Hz}$ exponential line broadening and Fourier transformation, spectra were automatically phase and baseline corrected. The chemical shift was calibrated to the TSP peak at 0.0 ppm. Peak assignments were done according to human metabolome database (HMDB; www.hmdb.ca), Chenomx NMR Suite 7.0 (Chenomx Inc., Alberta, Canada) and previously published data [45]. For cell extracts, the peak areas of lactate $(1.33 \mathrm{ppm})$, alanine $(1.48 \mathrm{ppm})$, glutamate (2.35 ppm) and glucose (4.64 ppm) and for culture mediums, the peak areas of lactate $(1.33 \mathrm{ppm})$ and glucose (4.64 ppm) were identified and fitted as Voigt curves by polynomial regression (PeakFit v 4.12; Systat Software Inc., Chicago, IL, USA). The correlation coefficient of the fit $\left(\mathrm{r}^{2}\right)$ for all spectra was $\geq 0.95$. Absolute concentration of individual metabolites were calculated with reference to peak area of the standard creatine solution and the cell extract samples were normalized to the protein content while culture medium samples were normalized to the concentration of TSP, according to the PULCON principle [46].

\section{ACKNOWLEDGMENTS}

We are grateful to Professor Suzanne Walker and Dr. Craig Thomas, who provided us with access to a novel OGT inhibitor (OSMI-1).

\section{FUNDING}

HMI is funded by the Norwegian Cancer Society. IGM is supported in Oslo by funding from the Norwegian Research Council, Helse Sor-Ost and the University of Oslo through the Centre for Molecular Medicine (Norway), which is a part of the Nordic EMBL (European Molecular Biology Laboratory) partnership. IGM holds a visiting scientist position with Cancer Research UK through the Cambridge Research Institute and a Senior Honorary Visiting Research Fellowship with Cambridge University through the Department of Oncology. IGM is supported in Belfast by the BelfastManchester Movember Centre of Excellence (CE013_2004), funded in partnership with Prostate Cancer UK. The NMR spectroscopy was performed at the MR Core Facility, Norwegian University of Science and Technology (NTNU). The MR core facility is funded by the Faculty of Medicine at NTNU and Central Norway Regional Health Authority. SESM is supported through National Institutes of Health R01 GM094263 granted to Suzanne Walker.

\section{CONFLICTS OF INTEREST}

No conflicts of interest to declare.

\section{REFERENCES}

1. Barfeld SJ, Itkonen HM, Urbanucci A and Mills IG. Androgen-regulated metabolism and biosynthesis in prostate cancer. Endocrine-related cancer. 2014; 21:T57-66.

2. Itkonen HM, Engedal N, Babaie E, Luhr M, Guldvik IJ, Minner S, Hohloch J, Tsourlakis MC, Schlomm T and Mills IG. UAP1 is overexpressed in prostate cancer and is protective against inhibitors of N-linked glycosylation. Oncogene. 2014.

3. Massie CE, Lynch A, Ramos-Montoya A, Boren J, Stark R, Fazli L, Warren A, Scott H, Madhu B, Sharma N, Bon H, Zecchini V, Smith DM, Denicola GM, Mathews N, Osborne $\mathrm{M}$, et al. The androgen receptor fuels prostate cancer by regulating central metabolism and biosynthesis. The EMBO journal. 2011; 30:2719-2733.

4. Heemers H, Vanderhoydonc F, Roskams T, Shechter I, Heyns W, Verhoeven G and Swinnen JV. Androgens stimulate coordinated lipogenic gene expression in normal target tissues in vivo. Molecular and cellular endocrinology. 
2003; 205:21-31.

5. Cantor JR and Sabatini DM. Cancer cell metabolism: one hallmark, many faces. Cancer discovery. 2012; 2:881-898.

6. Preston MA, Riis AH, Ehrenstein V, Breau RH, Batista JL, Olumi AF, Mucci LA, Adami HO and Sorensen HT. Metformin Use and Prostate Cancer Risk. European urology. 2014.

7. Ross SJ and Critchlow SE. Emerging approaches to target tumor metabolism. Current opinion in pharmacology. 2014; 17C:22-29.

8. Jia J, Zhu F, Ma X, Cao Z, Li Y and Chen YZ. Mechanisms of drug combinations: interaction and network perspectives. Nature reviews Drug discovery. 2009; 8:111-128.

9. Metallo CM and Vander Heiden MG. Metabolism strikes back: metabolic flux regulates cell signaling. Genes \& development. 2010; 24:2717-2722.

10. Hanover JA, Krause MW and Love DC. Bittersweet memories: linking metabolism to epigenetics through O-GlcNAcylation. Nature reviews Molecular cell biology. 2012; 13:312-321.

11. Vaidyanathan K, Durning $\mathrm{S}$ and Wells L. Functional O-GlcNAc modifications: implications in molecular regulation and pathophysiology. Critical reviews in biochemistry and molecular biology. 2014; 49:140-163.

12. Yi W, Clark PM, Mason DE, Keenan MC, Hill C, Goddard WA, 3rd, Peters EC, Driggers EM and Hsieh-Wilson LC. Phosphofructokinase 1 glycosylation regulates cell growth and metabolism. Science. 2012; 337:975-980.

13. Jang H, Kim TW, Yoon S, Choi SY, Kang TW, Kim SY, Kwon YW, Cho EJ and Youn HD. O-GlcNAc regulates pluripotency and reprogramming by directly acting on core components of the pluripotency network. Cell stem cell. 2012; 11:62-74.

14. Ranuncolo SM, Ghosh S, Hanover JA, Hart GW and Lewis BA. Evidence of the involvement of O-GlcNAc-modified human RNA polymerase II CTD in transcription in vitro and in vivo. The Journal of biological chemistry. 2012; 287:23549-23561.

15. Rozanski W, Krzeslak A, Forma E, Brys M, Blewniewski M, Wozniak P and Lipinski M. Prediction of bladder cancer based on urinary content of MGEA5 and OGT mRNA level. Clinical laboratory. 2012; 58:579-583.

16. Mi W, Gu Y, Han C, Liu H, Fan Q, Zhang X, Cong Q and $\mathrm{Yu}$ W. O-GlcNAcylation is a novel regulator of lung and colon cancer malignancy. Biochimica et biophysica acta. 2011; 1812:514-519.

17. Itkonen HM, Minner S, Guldvik IJ, Sandmann MJ, Tsourlakis MC, Berge V, Svindland A, Schlomm T and Mills IG. O-GlcNAc transferase integrates metabolic pathways to regulate the stability of c-MYC in human prostate cancer cells. Cancer research. 2013; 73:5277-5287.

18. Gross BJ, Kraybill BC and Walker S. Discovery of O-GlcNAc transferase inhibitors. Journal of the American Chemical Society. 2005; 127:14588-14589.
19. Ortiz-Meoz RF, Jiang J, Lazarus MB, Orman M, Janetzko J, Fan C, Duveau DY, Tan ZW, Thomas CJ and Walker S. A small molecule that inhibits OGT activity in cells. ACS chemical biology. 2015; 10:1392-1397.

20. Caldwell SA, Jackson SR, Shahriari KS, Lynch TP, Sethi G, Walker S, Vosseller K and Reginato MJ. Nutrient sensor $\mathrm{O}-\mathrm{GlcNAc}$ transferase regulates breast cancer tumorigenesis through targeting of the oncogenic transcription factor FoxM1. Oncogene. 2010; 29:2831-2842.

21. Lynch TP, Ferrer CM, Jackson SR, Shahriari KS, Vosseller $\mathrm{K}$ and Reginato MJ. Critical role of O-Linked beta-Nacetylglucosamine transferase in prostate cancer invasion, angiogenesis, and metastasis. The Journal of biological chemistry. 2012; 287:11070-11081.

22. Kazemi Z, Chang H, Haserodt S, McKen $\mathrm{C}$ and Zachara NE. O-linked beta-N-acetylglucosamine (O-GlcNAc) regulates stress-induced heat shock protein expression in a GSK-3beta-dependent manner. The Journal of biological chemistry. 2010; 285:39096-39107.

23. Chen S, Xu Y, Yuan X, Bubley GJ and Balk SP. Androgen receptor phosphorylation and stabilization in prostate cancer by cyclin-dependent kinase 1. Proceedings of the National Academy of Sciences of the United States of America. 2006; 103:15969-15974.

24. Malumbres M. Cyclin-dependent kinases. Genome Biol. 2014; 15:122.

25. Taylor BS, Schultz N, Hieronymus H, Gopalan A, Xiao Y, Carver BS, Arora VK, Kaushik P, Cerami E, Reva B, Antipin Y, Mitsiades N, Landers T, Dolgalev I, Major JE, Wilson $\mathrm{M}$, et al. Integrative genomic profiling of human prostate cancer. Cancer Cell. 2010; 18:11-22.

26. Barrientos A and Moraes CT. Titrating the effects of mitochondrial complex I impairment in the cell physiology. The Journal of biological chemistry. 1999; 274:1618816197.

27. Viollet B, Guigas B, Sanz Garcia N, Leclerc J, Foretz M and Andreelli F. Cellular and molecular mechanisms of metformin: an overview. Clinical science. 2012; 122:253270.

28. Adeva-Andany M, Lopez-Ojen M, Funcasta-Calderon R, Ameneiros-Rodriguez E, Donapetry-Garcia C, Vila-Altesor $\mathrm{M}$ and Rodriguez-Seijas J. Comprehensive review on lactate metabolism in human health. Mitochondrion. 2014; 17C:76-100.

29. Chou TY, Hart GW and Dang CV. c-Myc is glycosylated at threonine 58, a known phosphorylation site and a mutational hot spot in lymphomas. The Journal of biological chemistry. 1995; 270:18961-18965.

30. Bond MR and Hanover JA. A little sugar goes a long way: the cell biology of O-GlcNAc. The Journal of cell biology. 2015; 208:869-880.

31. Zhu Y, Liu TW, Madden Z, Yuzwa SA, Murray K, Cecioni S, Zachara N and Vocadlo DJ. Post-translational O-GlcNAcylation is essential for nuclear pore integrity 
and maintenance of the pore selectivity filter. Journal of molecular cell biology. 2015.

32. Mizuguchi-Hata C, Ogawa Y, Oka M and Yoneda Y. Quantitative regulation of nuclear pore complex proteins by O-GlcNAcylation. Biochimica et biophysica acta. 2013; 1833:2682-2689.

33. Laoukili J, Alvarez M, Meijer LA, Stahl M, Mohammed S, Kleij L, Heck AJ and Medema RH. Activation of FoxM1 during G2 requires cyclin A/Cdk-dependent relief of autorepression by the FoxM1 N-terminal domain. Molecular and cellular biology. 2008; 28:3076-3087.

34. Costa RH. FoxM1 dances with mitosis. Nature cell biology. 2005; 7:108-110.

35. Lazarus MB, Jiang J, Kapuria V, Bhuiyan T, Janetzko J, Zandberg WF, Vocadlo DJ, Herr W and Walker S. HCF1 is cleaved in the active site of O-GlcNAc transferase. Science. 2013; 342:1235-1239.

36. Zhong J, Martinez M, Sengupta S, Lee A, Wu X, Chaerkady R, Chatterjee A, O'Meally RN, Cole RN, Pandey A and Zachara NE. Quantitative phosphoproteomics reveals crosstalk between phosphorylation and O-GlcNAc in the DNA damage response pathway. Proteomics. 2015; 15:591607.

37. Vaz CV, Alves MG, Marques R, Moreira PI, Oliveira PF, Maia CJ and Socorro S. Androgen-responsive and nonresponsive prostate cancer cells present a distinct glycolytic metabolism profile. The international journal of biochemistry \& cell biology. 2012; 44:2077-2084.

38. Tessem MB, Swanson MG, Keshari KR, Albers MJ, Joun D, Tabatabai ZL, Simko JP, Shinohara K, Nelson SJ, Vigneron DB, Gribbestad IS and Kurhanewicz J. Evaluation of lactate and alanine as metabolic biomarkers of prostate cancer using 1H HR-MAS spectroscopy of biopsy tissues. Magnetic resonance in medicine. 2008; 60:510-516.

39. Nelson SJ, Kurhanewicz J, Vigneron DB, Larson PE, Harzstark AL, Ferrone M, van Criekinge M, Chang JW, Bok R, Park I, Reed G, Carvajal L, Small EJ, Munster P, Weinberg VK, Ardenkjaer-Larsen JH, et al. Metabolic imaging of patients with prostate cancer using hyperpolarized [1-(1)(3)C]pyruvate. Science translational medicine. 2013; 5:198ra108.

40. Beuster G, Zarse K, Kaleta C, Thierbach R, Kiehntopf M, Steinberg P, Schuster S and Ristow M. Inhibition of alanine aminotransferase in silico and in vivo promotes mitochondrial metabolism to impair malignant growth. The Journal of biological chemistry. 2011; 286:22323-22330.

41. Caminero JA, Sotgiu G, Zumla A and Migliori GB. Best drug treatment for multidrug-resistant and extensively drugresistant tuberculosis. The Lancet Infectious diseases. 2010; 10:621-629.

42. Musana KA, Yale SH and Abdulkarim AS. Tests of liver injury. Clinical medicine \& research. 2004; 2:129-131.

43. Hart GW. Three Decades of Research on O-GlcNAcylation - A Major Nutrient Sensor That Regulates Signaling,
Transcription and Cellular Metabolism. Frontiers in endocrinology. 2014; 5:183.

44. Austdal M, Skrastad RB, Gundersen AS, Austgulen R, Iversen $\mathrm{AC}$ and Bathen TF. Metabolomic biomarkers in serum and urine in women with preeclampsia. PloS one. 2014; 9:e91923.

45. Sitter B, Bathen TF, Tessem M-B and Gribbestad IS. High-resolution magic angle spinning (HR MAS) MR spectroscopy in metabolic characterization of human cancer. Progress in Nuclear Magnetic Resonance Spectroscopy. 2009; 54:239-254.

46. Wider G and Dreier L. Measuring protein concentrations by NMR spectroscopy. Journal of the American Chemical Society. 2006; 128:2571-2576. 